\title{
¿ES LA ORIENTACIÓN SEXUAL REALMENTE ESTABLE?: DIFERENCIAS DE GÉNERO
}

\author{
Juan Enrique Nebot-Garcia \\ Grupo Salusex. Departamento de Psicología Básica, Clínica y Psicobiología \\ Universitat Jaume I, Castellón, España \\ Rafael Ballester-Arnal \\ Grupo Salusex. Departamento de Psicología Básica, Clínica y Psicobiología \\ Universitat Jaume I, Castellón, España \\ Cristina Giménez-García \\ Grupo Salusex. Departamento de Psicología Básica, Clínica y Psicobiología \\ Universitat Jaume I, Castellón, España \\ Estefanía Ruiz-Palomino \\ Grupo Salusex. Departamento de Psicología Básica, Clínica y Psicobiología \\ Universitat Jaume I, Castellón, España \\ Naiara Martínez-Gómez \\ Grupo Salusex. Departamento de Psicología Básica, Clínica y Psicobiología \\ Universitat Jaume I, Castellón, España
}

Recepción Artículo: 26 febrero 2020

Admisión Evaluación: 4 marzo 2020

Informe Evaluador 1: 13 marzo 2020

Informe Evaluador 2: 15 Marzo 2020

Aprobación Publicación: 20 abril 2020

Este trabajo ha sido posible en parte gracias a la ayuda para el proyecto de investigación UJI-B2018-42 y la ayuda PREDOC/2017/45 de la Universitat Jaume I de Castellón.

\section{RESUMEN}

Introducción: Tradicionalmente la sexualidad ha sido conceptualizada desde una perspectiva conservadora, considerando la orientación sexual como un constructo hermético e inflexible. Estas creencias conservadoras, que se han traducido en actitudes homófobas, parecen ser más notorias en los hombres, posiblemente por el peso que tiene en ellos el concepto de masculinidad de los roles tradicionales de género.

Objetivo: Conocer la edad de definición de la orientación sexual, hasta qué punto ésta es una dimensión estable a lo largo del ciclo vital, la valoración de las etiquetas tradicionales y la posible existencia de diferencias de género en estas variables.

Metodología: 2924 españoles de entre 18 y 73 años ( $M=26,29$; DT=9,10) participaron en el presente estudio, siendo el 32,9\% hombres y el 67,1\% mujeres. En cuanto a la orientación sexual, el 77,8\% se identificaban como heterosexuales, el 9,6\% como homosexuales y el $12,6 \%$ como bisexuales. Todos ellos cumplimentaron un cuestionario ad hoc sobre orientación sexual, toma de conciencia y evolución de su autodefinición sexual, así como valoración de las etiquetas tradicionales.

Resultados: La muestra evaluada definió su orientación sexual a los 12,2 años de edad (DT=4,70). En fun- 


\section{¿ES LA ORIENTACIÓN SEXUAL REALMENTE ESTABLE?: DIFERENCIAS DE GÉNERO}

ción del género, las mujeres la definen a los 12,5 años (DT=4,41) y los hombres a los 11,5 (DT=5,18), observándose diferencias significativas ( $t=4,37 ; p<0,001)$. En cuanto a su evolución, el 11,5\% de los hombres y el 15,3\% de las mujeres (chi ${ }^{2}=7,46 ; p=0,006$ ) ha experimentado cambios en su orientación sexual a lo largo de su vida. Por otra parte, un $58,6 \%$ de los hombres y un $72,4 \%$ de las mujeres consideran que las etiquetas tradicionales sobre orientación sexual (heterosexual, bisexual y homosexual) no representan fielmente a la sociedad actual $\left(c^{2}{ }^{2}=56,19 ; p<0,001\right)$. Y, por último, se observa que las personas que habían tardado más en definir su orientación sexual son aquellas que habían experimentado cambios en su orientación ( $t=26,52 ; p<0,001)$ y consideraban que las categorías tradicionales de orientación no representaban fielmente a la sociedad actual ( $t=3,77 ; p<0,001)$.

Conclusiones: Estos resultados muestran la fluidez y flexibilidad existente en la vivencia de la orientación sexual. Un porcentaje importante de la población parece reflexionar sobre su sexualidad y cuestionar las categorías tradicionales, lo que podría atrasar la definición estable de la propia orientación sexual, siendo este efecto más notorio en las mujeres. Estos hallazgos deben ser considerados cuando se conceptualice la orientación sexual, no solo como una dimensión continua, sino también que evoluciona a lo largo del ciclo vital.

Palabras clave: orientación sexual; toma de conciencia; fluidez sexual; género; ciclo vital

\section{ABSTRACT}

Is sexual orientation really stable?: gender differences. Introduction: Traditionally, sexuality has been conceptualized from a conservative perspective, considering sexual orientation as a hermetic and inflexible construct. These conservative beliefs, which have been translated into homophobic attitudes, seem to be more noticeable in men, possibly due to the importance that the concept of masculinity in traditional gender roles has in them.

Objective: To know the age of definition of sexual orientation, if sexual orientation is a stable dimension throughout the life cycle, the assessment of traditional labels and the possible existence of gender differences in these variables.

Methodology: 2924 Spaniards between 18 and 73 years old ( $M=26.29$; SD=9.10) participated in the present study, with $32.9 \%$ men and $67.1 \%$ women. Regarding sexual orientation, $77.8 \%$ identified as heterosexual, $9.6 \%$ as homosexual and $12.6 \%$ as bisexual. All of them completed an ad hoc questionnaire on sexual orientation, awareness and evolution of their sexual self-definition, as well as an assessment of traditional labels.

Results: The evaluated sample defined their sexual orientation at 12.2 years of age (SD=4.70). Based on gender, women defined it at 12.5 years old $(S D=4.41)$ and men at 11.5 year old $(S D=5.18)$, with significant differences $(t=4.37 ; p<0.001)$. Regarding their evolution, $11.5 \%$ of men and $15.3 \%$ of women ( $c h i^{2}=7.46 ; p=0.006$ ) have experienced changes in their sexual orientation throughout their lives. On the other hand, $58.6 \%$ of men and $72.4 \%$ of women consider that traditional labels on sexual orientation (heterosexual, bisexual and homosexual) do not accurately represent today's society $\left(c h^{2}=56.19 ; p<0.001\right)$. And, finally, it is observed that the people who had defined later their sexual orientation were those who had experienced changes in it $(t=26.52 ; p<0.001)$ and who had considered that the traditional categories of orientation did not accurately represent to current society $(\mathrm{t}=3.77 ; \mathrm{p}<0.001)$.

Conclusions: These results show the fluidity and the flexibility existing in the experience of sexual orientation. A significant percentage of the population seems to reflect on their sexuality and question traditional categories, which could delay the stable definition of their sexual orientation. This effect is more noticeable in women. These findings should be considered when conceptualizing sexual orientation, not only as a continuous dimension, but also as construct that evolves throughout the life cycle.

Keywords: sexual orientation; awareness; sexual fluidity; gender; life span

\section{INTRODUCCIÓN}

Tradicionalmente la orientación sexual ha sido concebida como un constructo binario, con categorías cerradas y estables en el tiempo, donde solo existían dos posibilidades: heterosexualidad y homosexualidad, y evi- 
dentemente, solo la primera era considerada normal y saludable (Callis, 2014; Carroll, 2015). No fue hasta los años cincuenta, con la llegada de los estudios de Kinsey (Kinsey, Pomeroy, \& Martin, 1948; Kinsey, Pomeroy, Martin, \& Gebhard, 1953), cuando se flexibilizaron estos conceptos y se empezó a entender la orientación sexual como una dimensión con diferentes posiciones intermedias, dando visibilidad a orientaciones sexuales como la bisexualidad y a distintos grados intermedios Años más tarde, en 1978, Fritz Klein (Klein, 2012) fue un poco más allá y abordó este constructo de forma multidimensional, considerando que la orientación sexual estaba formada por diferentes dimensiones, como la conducta sexual, la atracción sexual, las fantasías sexuales, etc. Además, afirmó que estas dimensiones no eran estables, sino que fluctuaban en función del tiempo.

Pese a esta concepción más flexible de la orientación sexual, en la sociedad sigue imperando la heteronormatividad, donde lo natural y lo normalizado son las relaciones heterosexuales (Herz \& Johansson, 2015). Es por eso que, muchas veces, aquellas personas con una orientación diferente a la heterosexual terminan sufriendo discriminación (Digoix et al., 2016). Sin embargo, no es solo el colectivo LGTBI (Lesbianas, Gays, Transexuales, Bisexuales e Intersexuales) quien sufre esta presión heteronormativa, sino que los propios heterosexuales ven sus conductas condicionadas al tener que ajustarse a lo que socialmente se espera de ellos como heterosexuales, provocando malestar en aquellas personas que muestran conductas que se alejan de la norma (Bosson, PrewittFreilino, \& Taylor, 2005).

De una manera u otra, esta presión heteronormativa repercute e influye en la conformación de la identidad psicosexual de las personas, que es el proceso mediante el cual un individuo toma conciencia de sus deseos, atracciones y sentimientos, y empieza a integrarlos dentro de la categoría de orientación sexual con la que más se identifica (Soriano, 2004). Pese a que gran parte de la población heterosexual no es consciente de todo este proceso porque coincide, prácticamente, con los modelos sociales y los cánones esperados, existe un sector de la población heterosexual que, igual que el colectivo LGTBI, pasa por un cuestionamiento de su sexualidad antes de definirse (Morgan, Steiner, \& Thompson 2010; Morgan \& Thompson 2011).

Esta influencia normativa parece repercutir de manera diferencial en hombres y mujeres. Los hombres se permiten menos explorar su orientación sexual, posiblemente debido a los roles de género y el concepto tradicional de masculinidad (Cornejo, 2015; Parrott, 2009; Parrott \& Zeichner, 2008) que castiga más los comportamientos homosexuales (Petersen \& Hyde, 2011; Poteat \& Anderson, 2012) y los femeninos en los hombres (Carnaghi, Maass, \& Fasoli, 2011; Glick, Gangl, Gibb, Klumpner, \& Weinberg, 2007). En cambio, en las mujeres estos comportamientos homosexuales suelen ser fomentados por los hombres, ya que se perciben las conductas lésbicas como algo excitante en sus fantasías sexuales (Yost \& McCarthy, 2012).

Por todo ello, el objetivo del presente trabajo es analizar si existen diferencias de género en la edad de definición de la orientación sexual, en el porcentaje de personas que ha experimentado cambios en su orientación sexual y en la valoración que hacen de las etiquetas tradicionales. Además, se pretende analizar si, el hecho de experimentar cambios en la orientación o mostrar un rechazo hacia las categorías tradicionales repercute en la edad de toma de conciencia. En cuanto a las hipótesis, se espera que: 1) Las mujeres definirán su orientación sexual a una edad más tardía, en comparación con los hombres; 2) Un mayor número de mujeres habrá experimentado cambios en su orientación sexual, en comparación con los hombres; 3) Las mujeres mostrarán más rechazo a las categorías tradicionales de orientación sexual, en comparación con los hombres; 4) Las personas que han experimentado cambios en su orientación y muestran rechazo hacia las categorías tradicionales de orientación sexual tardarán más en definir su orientación sexual.

\section{PARTICIPANTES}

A partir de un muestreo aleatorio simple, se seleccionaron un total de 2924 españoles de entre 18 y 73 años ( $M=26,29 ; D . T=9,10)$, siendo el 32,9\% hombres y el $67,1 \%$ mujeres. En cuanto a la orientación sexual, el $77,8 \%$ se definían como heterosexuales, el 9,6\% como homosexuales y el 12,6\% como bisexuales. Los criterios de inclusión eran: ser mayor de 18 años, ser hombre o mujer, autodefinirse como heterosexual, homosexual o bisexual, y residir en España. 


\section{¿ES LA ORIENTACIÓN SEXUAL REALMENTE ESTABLE?: DIFERENCIAS DE GÉNERO}

\section{INSTRUMENTOS DE EVALUACIÓN}

Todos Ios participantes cumplimentaron un cuestionario ad hoc de 94 ítems, realizado por nuestro grupo Salusex-Unisexsida, sobre orientación sexual y vivencias relacionadas. Pese a que esta investigación formaba parte de un proyecto más amplio, para este estudio solo se utilizaron tres ítems: una pregunta abierta para averiguar «aproximadamente, ¿a qué edad definiste tu actual orientación sexual?», y dos preguntas dicotómicas que debían responderse con «Sí» 0 «No», «a lo largo de los años, ¿has cambiado tu orientación sexual?» y «¿consideras que las etiquetas tradicionales sobre orientación sexual (heterosexual, bisexual y homosexual) representan fielmente a la sociedad actual?».

\section{PROCEDIMIENTO}

Con la intención de llegar a población de toda la geografía española, se realizó un anuncio de la investigación y se difundió en varios grupos generalistas de redes sociales. Una vez clicaban en el anuncio, se les informaba del carácter anónimo, voluntario y confidencial de la investigación y se les pedía el consentimiento informado. A continuación, accedían al cuestionario on-line desde sus dispositivos. Esta investigación contaba con la aprobación de la Comisión Deontológica de la Universitat Jaume I (Castellón, España) y, en todo momento, se siguieron los principios éticos de la Declaración de Helsinki y se tomaron las medidas oportunas que marca la legislación más reciente para cumplir con la protección de datos.

\section{ANALISIS ESTADÍSTICO}

En primer lugar, se utilizó el programa estadístico SPSS versión 25 para realizar análisis descriptivos de las principales variables sociodemográficas. A continuación, para analizar las diferencias en el promedio de edad de toma de conciencia, se utilizó la t de Student; y para conocer las prevalencias y analizar las diferencias de cambio en la orientación sexual y la valoración sobre las categorías tradicionales, se realizaron tablas de contingencias y pruebas de Chi cuadrado.

\section{RESULTADOS}

En términos generales, la muestra evaluada definió su orientación sexual en torno a los 12,16 años de edad (DT=4,70). Si tenemos en cuenta el género, los hombres la definen a los 11,55 años ( $D T=5,18)$ y las mujeres a los 12,47 años (DT=4,41), siendo las diferencias significativas estadísticamente $(t=4,73 ; p<0,001)$. Si los separamos por orientación sexual y género (véase Tabla 1), se observa que las personas bisexuales son las que más tardan en definir su orientación sexual, seguidas de las homosexuales y, en último término, las heterosexuales. Hay que destacar las importantes diferencias a este respecto con una demora de cuatro años en los/as homosexuales y de seis a ocho en los/as bisexuales respecto a la edad promedio de toma de conciencia de los heterosexuales. Dentro de una misma orientación sexual, solo se obtienen diferencias significativas entre hombres y mujeres en la categoría de heterosexual.

Tabla 1. Análisis de las diferencias en la edad de autodefinición de la orientación sexual, en función del género y de la orientación sexual.

\begin{tabular}{lccc}
\hline & Hombre & Mujer & \\
\cline { 2 - 3 } & $\mathrm{M}(\mathrm{DT})$ & $\mathrm{M}(\mathrm{DT})$ & $\mathrm{t}(\mathrm{p})$ \\
\hline Heterosexuales & $10,19(4,35)$ & $11,36(3,91)$ & $6,19(<0,001)$ \\
Bisexuales & $18,49(6,22)$ & $17,17(3,35)$ & $1,87(0,064)$ \\
Homosexuales & $14,48(3,83)$ & $15,22(3,96)$ & $1,58(0,114)$ \\
\hline
\end{tabular}

A lo largo de los años, un 14\% de la muestra evaluada había experimentado cambios en su orientación sexual. En función del género, un 11,5\% de hombres y un 15,2\% de mujeres había cambiado de orientación 
sexual, siendo las diferencias estadísticamente significativas $\left(\mathrm{Chi}^{2}=7,33 ; \mathrm{p}=0,007\right)$. Si los dividimos por género y orientación sexual (véase Tabla 2), se observa una tendencia mayor de las mujeres a experimentar cambios en su orientación sexual, pero las diferencias de género solo fueron significativas entre las personas homosexuales. En cuanto a su orientación sexual, las personas bisexuales son las que más cambios muestran en su orientación sexual, en segundo lugar, estarían las personas homosexuales y, con muy poca prevalencia, las heterosexuales. La diferencia entre las distintas orientaciones es muy importante, como se puede apreciar en la tabla 2.

Tabla 2. Análisis diferencial del porcentaje de participantes que ha experimentado cambios en su orientación sexual, en función del género y de la orientación sexual.

\begin{tabular}{lccc} 
& Hombre & Mujer & \\
\cline { 2 - 3 } & $\%$ & $\%$ & $\mathrm{Chi}^{2}(\mathrm{p})$ \\
\hline Heterosexuales & 1,6 & 2,1 & $0,52(0,471)$ \\
Bisexuales & 57,1 & 68,4 & $3,67(0,067)$ \\
Homosexuales & 35,7 & 52,6 & $8,10(0,004)$ \\
\hline
\end{tabular}

Por otro lado, el $67,9 \%$ de la población considera que las etiquetas tradicionales de "heterosexual", "bisexual" y "homosexual" no representan fielmente a la sociedad actual. Más concretamente, un 58,6\% de hombres y un $72,4 \%$ de mujeres piensan en esta línea, siendo las diferencias estadísticamente significativas ( $C h{ }^{2}=55,92$; $p<0,001$ ). Si realizamos un análisis en función de su orientación sexual y su género (véase Tabla 3), observamos que las mujeres son las que más rechazo muestran ante estas categorías tradicionales y cómo los hombres concretamente heterosexuales se mantienen a mucha distancia de todo el resto de participantes. Sin embargo, estas diferencias de género solo son significativas en las personas heterosexuales y en las homosexuales. Si solamente tenemos en cuenta su orientación sexual, las personas homosexuales y bisexuales son las que más en contra están ante estas etiquetas.

Tabla 3. Análisis diferencial del porcentaje de participantes que están en desacuerdo con las etiquetas tradicionales de orientación sexual, en función del género y de la orientación sexual.

\begin{tabular}{lccc}
\hline & Hombre & Mujer & \\
\cline { 2 - 3 } & $\%$ & $\%$ & Chi $^{2}(\mathrm{p})$ \\
\hline Heterosexuales & 54,7 & 70,7 & $56,53(<0,001)$ \\
Bisexuales & 71,4 & 76,5 & $0,89(0,344)$ \\
Homosexuales & 71,3 & 82,5 & $4,88(0,027)$ \\
\hline
\end{tabular}

Por último, se observa que las personas que han tardado más en definir su orientación sexual son aquellas que han experimentado cambios en su orientación y consideran que las categorías tradicionales de orientación sexual (heterosexual, bisexual y homosexual) no representan fielmente a la sociedad actual, siendo las diferencias estadísticamente significativas en ambos casos (véase Tabla 4). 


\section{¿ES LA ORIENTACIÓN SEXUAL REALMENTE ESTABLE?: DIFERENCIAS DE GÉNERO}

Tabla 4. Análisis diferencial de la edad de autodefinición de la orientación sexual en función de si se ha producido cambio en la etiqueta o no, y en función de si se está a favor o en contra de las categorías tradicionales.

\begin{tabular}{ccc} 
& $\begin{array}{c}\text { Edad } \\
\text { M (DT) }\end{array}$ & $\mathrm{t}(\mathrm{p})$ \\
\hline Cambio Orientación Sexual & & \\
$\quad$ Sí & $17,12(4,28)$ & 26,52 \\
No & $11,36(4,04)$ & $(<0,001)$ \\
Categorías tradicionales & & \\
$\quad$ A favor & $11,68(4,82)$ & 3,77 \\
En contra & $12,39(4,62)$ & $(<0,001)$ \\
\hline
\end{tabular}

\section{CONCLUSIONES}

El objetivo del presente trabajo era conocer la edad de definición de la orientación sexual, su estabilidad temporal, la valoración de las etiquetas con las que tradicionalmente se ha clasificado a las personas en función de su orientación sexual, y determinar si existían diferencias de género en estas variables. Además, se pretendía analizar si, el hecho de experimentar cambios en la orientación y mostrar un rechazo hacia las categorías tradicionales repercutía en la edad de toma de conciencia.

En cuanto a la edad de toma de conciencia, tal y como se observa en otros estudios (Petersen \& Hyde, 2011), las mujeres definen su orientación sexual a una edad más tardía, lo que podría tener relación con que las mujeres se cuestionen su orientación sexual más que los hombres (Morgan et al., 2010; Morgan \& Thompson, 2011); por tanto, se cumple la primera hipótesis. No obstante, cuando se separa por orientación sexual, las diferencias de género solo son significativas en población heterosexual. Esto podría deberse al hecho de que, la presión social heteronormativa y los roles de género tradicionales afecten sustancialmente más a hombres heterosexuales (Cornejo, 2015; Parrott, 2009; Parrott \& Zeichner, 2008), pero no tanto a hombres de otras orientaciones sexuales. Por otra parte, las personas heterosexuales son las que más pronto definen su orientación sexual, lo que tendría sentido si tenemos en cuenta que no necesitarían cuestionarse su sexualidad, ya que la sociedad espera que sean heterosexuales (Herz \& Johansson, 2015). Es posible que en muchos casos se pueda producir el hecho de que la presión social hacia la heterosexualidad haga que, especialmente los hombres a los que se les permite en menor medida apartarse del rol masculino tradicional (Cornejo, 2015; Parrott, 2009; Parrott \& Zeichner, 2008), se autoidentifiquen más tempranamente en su orientación heterosexual sin haberse tomado el tiempo necesario para explorar lo que realmente desean y les excita. En cambio, las personas bisexuales y homosexuales, al tener que luchar con lo esperado y replantearse qué es exactamente lo que sienten y desean, tardarían más tiempo en definirse. Este proceso es más tardío, si cabe, en las personas bisexuales y podría deberse a que los individuos, cuando empiezan a sentir que no son heterosexuales, se muevan al extremo completamente opuesto (dentro de esa concepción social dicotómica de los deseos), y necesitarían de más tiempo para situarse de nuevo en posiciones intermedias. También puede ser que les cueste más identificarse como bisexuales dada la discriminación y los prejuicios existentes hacia toda la comunidad bisexual (Yost \& Thomas, 2012). 0, en tercer lugar, que la etiqueta "bisexual" no sea vista hasta tarde porque más que una etiqueta en sí misma se perciba como la ausencia de las otras dos que ocupan los extremos. De hecho, en la literatura científica se puede encontrar el debate acerca de si existe realmente una identidad bisexual o más bien comportamientos bisexuales (Gooß, 2008).

En cuanto a la segunda hipótesis, también se cumple, ya que se observa que un porcentaje mayor de mujeres ha experimentado cambios en su orientación sexual, en comparación con los hombres. Estas diferencias también se encontraron en otras investigaciones similares (Diamond, 2016; Katz-Wise, 2015; Morgan et al., 2010; 
Morgan \& Thompson, 2011). No obstante, si se tiene en cuenta la orientación sexual, las diferencias de género solo fueron significativas entre la población homosexual. Esto podría guardar relación con la fluidez sexual de la que hablaba Diamond (2008), que sostenía que muchas mujeres, descubren y manifiestan sus deseos lésbicos en edades más tardías, por lo que, en etapas iniciales de su descubrimiento sexual, podrían haberse identificado como heterosexuales. 0 con el hecho de que simplemente las mujeres tengan esquemas sexuales y de género menos rígidos que los hombres. En términos generales, las personas bisexuales son las que mayores cambios han experimentado, lo que iría en línea con lo expuesto anteriormente. Puede que, en primera estancia se hayan situado en uno de los extremos (heterosexual u homosexual), y después hayan descubierto que sus sentimientos y atracciones se correspondían más con una persona bisexual.

En cuanto a las etiquetas tradicionales, las mujeres mostraron más rechazo a las categorías tradicionales de orientación sexual, por lo que la tercera hipótesis también se cumple. Este rechazo hacia las etiquetas tradicionales de orientación sexual podría guardar relación con el hecho de que las mujeres muestran una mayor aceptación hacia toda la diversidad sexual, en comparación con los hombres (García-Barba, Serrano-Moragón, Camañes-Martorell, Ruiz-Palomino, \& Ballester-Arnal, 2018; Norton \& Herek, 2013; Petersen \& Hyde, 2011; Yost \& Thomas, 2012). De hecho, su apertura es tal que en un estudio se observó que las mujeres tienen más probabilidad de identificarse con identidades queer o pansexuales que los hombres (Morandini, Blaszczynski, \& DarNimrod, 2017). En cuanto a la orientación sexual, se observa que las personas bisexuales y homosexuales son las que mayor desacuerdo muestran con estas categorías, lo que podría guardar relación con el hecho de que el colectivo LGTBI, para identificarse como tal, ha tenido que reflexionar sobre su sexualidad e ir en contra de lo que se esperaba (Soriano, 2004), por lo que es más fácil que se muestren críticos con estas etiquetas tradicionales.

Por último, se ha observado que las personas que han experimentado cambios en su orientación o que muestran rechazo hacia las categorías tradicionales definen su orientación sexual a una edad más tardía, lo que verificaría la última hipótesis. Estos cambios y el espíritu crítico hacia lo sexualmente tradicional podrían ser sinónimos de una mayor apertura y una mayor reflexión, lo que implicaría una inversión de tiempo hasta llegar a concluir qué orientación sexual nos define mejor.

En términos generales, se observa que las mujeres se permiten reflexionar más sobre su sexualidad y se rebelan más respecto a lo que tradicionalmente se espera de ellas. En cambio, los hombres siguen más las concepciones tradicionales y las normas sociales. Estos comportamientos, como se ha dicho, podrían deberse a la rigidez de los roles tradicionales de género (Carnaghi, Maass, \& Fasoli, 2011; Cornejo, 2015; Parrott, 2009; Parrott \& Zeichner, 2008) que, en este aspecto, afectan de forma más directa a los hombres. El temor a la pérdida de la masculinidad, entendida según la tradición, resulta ser una losa para el desarrollo pleno de la sexualidad de muchos hombres.

Si bien esta investigación aporta información relevante sobre los procesos evolutivos y madurativos de la orientación sexual, no hay que dejar de lado las limitaciones que presenta el estudio, como el modesto tamaño muestral de algunas orientaciones sexuales, que sería adecuado aumentar para poder obtener resultados más concluyentes y representativos. Del mismo modo, para futuros estudios también podría incluirse la variable generacional para determinar si el proceso de identificación psicosexual es igual en diferentes épocas.

Así pues, cabría tener en cuenta todos estos resultados para elaborar programas de educación sexual y acercamientos terapéuticos que muestren la realidad sobre las diferentes orientaciones sexuales y sus características, dando visibilidad a su carácter flexible y dimensional. Todo ello serviría para romper los estereotipos asociados a las etiquetas sexuales y reduciría el malestar que sienten las personas al sentir que están rompiendo con las normas sociales y lo que se espera de ellos (Bosson et al., 2005; Soriano, 2004). 


\section{¿ES LA ORIENTACIÓN SEXUAL REALMENTE ESTABLE?: DIFERENCIAS DE GÉNERO}

\section{REFERENCIAS BIBLIOGRÁFICAS}

Bosson, J. K., Prewitt-Freilino, J. L., \& Taylor, J. N. (2005). Role rigidity: A problem of identity misclassification? Journal of Personality and Social Psychology, 89(4), 552-565.

Callis, A. S. (2014). Bisexual, pansexual, queer: Non-binary identities and the sexual borderlands. Sexualities, 17(1-2), 63-80.

Carnaghi, A., Maass, A., \& Fasoli, F. (2011). Enhancing masculinity by slandering homosexuals: The role of homophobic epithets in heterosexual gender identity. Personality and Social Psychology Bulletin, 37(12), 1655-1665.

Carroll, J. L. (2015). Sexuality now: Embracing diversity (5th edition). EE UU: Cengage Learning.

Cornejo, J. (2015). Componentes ideológicos de la homofobia. Límite. Revista de Filosofía y Psicología, 7(26), $85-106$.

Diamond, Lisa M. (2008). Sexual fluidity: Understanding women's love and desire. Cambridge, MA: Harvard University Press.

Diamond, L. M. (2016). Sexual fluidity in male and females. Current Sexual Health Reports, 8(4), 249-256.

Digoix, M., Franchi, M., Pichardo Galán, J. I., Selmi, G., de Stéfano Barbero, M., Thibeaud, M. \& Vela, J. A. M. (2016). Sexual orientation, family and kinship in France, Iceland, Italy and Spain. Families and Societies Working Paper 54(2016). Recuperado el 13 Febrero, 2020, de: http://www.familiesandsocieties.eu/wp-content/uploads/2016/08/WP54Digoixetal2016.pdf

García-Barba, M., Serrano-Moragón, N., Camañes-Martorell, J., Ruiz-Palomino, E., \& Ballester-Arnal, R. (2018). Actitudes hacia la transexualidad en jóvenes. Revista Àgora de Salut, 5, 11-19.

Glick, P., Gangl, C., Gibb, S., Klumpner, S., \& Weinberg, E. (2007). Defensive reactions to masculinity threat: More negative affect toward effeminate (but not masculine) gay men. Sex Roles, 57(1-2), 55-59.

Gooß, U. (2008). Concepts of bisexuality. Journal of Bisexuality, 8(1-2), 9-23.

Herz, M. \& Johansson, T. (2015). The normativity of the concept of heteronormativity. Journal of Homosexuality, 62(8), 1009-1020.

Katz-Wise, S. L. (2015). Sexual fluidity in young adult women and men: Associations with sexual orientation and sexual identity development. Psychology \& Sexuality, 6(2), 189-208.

Kinsey, A., Pomeroy, W., \& Martin, C. (1948). Sexual behavior in the human male. Philadelphia: Saunders Company.

Kinsey, A., Pomeroy, W., Martin, C., \& Gebhard, P. H. (1953). Sexual behavior in the human female. Indiana University Press.

Klein, F. (2012). La opción bisexual: Segunda edición. California, USA: American Institute of Bisexuality.

Morandini, J. S., Blaszczynski, A., \& Dar-Nimrod, I. (2017). Who adopts queer and pansexual sexual identities?. The Journal of Sex Research, 54(7), 911-922.

Morgan, E. M., Steiner, M. G., \& Thompson, E. M. (2010). Processes of sexual orientation questioning among heterosexual men. Men and Masculinities, 12(4), 425-443.

Morgan, E. M., \& Thompson, E. M. (2011). Processes of sexual orientation questioning among heterosexual women. Journal of Sex Research, 48(1), 16-28.

Norton, A. T., \& Herek, G. M. (2013). Heterosexuals' attitudes toward transgender people: Findings from a national probability sample of US adults. Sex roles, 68(11-12), 738-753.

Parrott, D. J. (2009). Aggression toward gay men as gender role enforcement: Effects of male role norms, sexual prejudice, and masculine gender role stress. Journal of Personality, 77(4), 1137-1166.

Parrott, D. J., \& Zeichner, A. (2008). Determinants of anger and physical aggression based on sexual orientation: An experimental examination of hypermasculinity and exposure to male gender role violations. Archives of Sexual Behavior, 37(6), 891-901.

Petersen, J. L., \& Hyde, J. S. (2011). Gender differences in sexual attitudes and behaviors: A review of meta-ana- 
Iytic results and large datasets. Journal of Sex Research, 48(2-3), 149-165.

Poteat, V. P., \& Anderson, C. J. (2012). Developmental changes in sexual prejudice from early to late adolescence: the effects of gender, race, and ideology on different patterns of change. Developmental psychology, 48(5), 1403-1415.

Soriano, S. (2004). Cómo se vive la homosexualidad y el lesbianismo (2ª edición). Salamanca: Amarú Ediciones.

Yost, M. R., \& McCarthy, L. (2012). Girls gone wild? Heterosexual women's same-sex encounters at college parties. Psychology of Women Quarterly, 36(1), 7-24.

Yost, M. R., \& Thomas, G. D. (2012). Gender and binegativity: Men's and women's attitudes toward male and female bisexuals. Archives of Sexual Behavior, 41(3), 691-702. 
